\title{
A New Approach to Intelligent Model Based Predictive Control Scheme
}

\author{
A. H. MAZINAN ${ }^{1}$, M. F. KAZEMI ${ }^{2}$ \\ ${ }^{1}$ Electrical Engineering Department, Islamic Azad University (IAU), South Tehran Branch, Tehran, Iran \\ ${ }^{2}$ Electrical Engineering Department, Islamic Azad University (IAU), Lahijan Branch, Lahijan, Iran \\ Emails:mazinan@azad.ac.ir,mfkazemi@iau-lahijan.ac.ir
}

\begin{abstract}
This paper describes a new approach to intelligent model based predictive control scheme for deriving a complex system. In the control scheme presented, the main problem of the linear model based predictive control theory in dealing with severe nonlinear and time variant systems is thoroughly solved. In fact, this theory could appropriately be improved to a perfect approach for handling all complex systems, provided that they are firstly taken into consideration in line with the outcomes presented. This control scheme is organized based on a multi-fuzzy-based predictive control approach as well as a multi-fuzzy-based predictive model approach, while an intelligent decision mechanism system (IDMS) is used to identify the best fuzzy-based predictive model approach and the corresponding fuzzy-based predictive control approach, at each instant of time. In order to demonstrate the validity of the proposed control scheme, the single linear model based generalized predictive control scheme is used as a benchmark approach. At last, the appropriate tracking performance of the proposed control scheme is easily outperformed in comparison with previous one.
\end{abstract}

Keywords: multi-fuzzy-based predictive control approach, multi-fuzzy-based predictive model approach, intelligent decision mechanism system

\section{Introduction}

Nowadays, the linear model based predictive control theory has been widely used in so many applications, in the past two decades. This control theory guarantees that the future error signals will be approached to zero by optimizing the specific cost function, if the linear model approximation of a complex system could accurately be identified. Based on the matter presented here, a complex system cannot actually be dealt with, while we are using the linear model based predictive control theory. In order to improve the present results, a new control scheme is now proposed to derive a severe nonlinear and time variant system in accordance with this control theory. The advantage of the proposed scheme is to realize an intelligent control method in the absence of any linear models of the system. In the control scheme proposed, a multi-fuzzy-based predictive model-predictive control approach so called MFPMPC scheme in this paper is organized to cope with an industrial tubular heat exchanger system as a severe nonlinear and time variant system. To realize the control scheme, at first, fuzzy-based predictive model approach and subsequently fuzzy-based predictive control approach need to be correspondingly realized at a specific operating point of the system. After that, the multi-fuzzy-based predictive model approach and then the multi-fuzzy-based predictive control approach are correspondingly implemented on the system to cover all the operating points. The rest of the proposed control algorithm is based on an intelligent decision mechanism system (IDMS) realization to choose the best fuzzy-based model approach and also the corresponding fuzzy-based predictive control approach, at each instant of time. In fact, the system behavior is fully covered via the IDMS, provided that the multifuzzy-based predictive model approach as well as the multi-fuzzy-based predictive control approach is suitably organized. It means that the best fuzzy-based predictive model approach and therefore the best fuzzy-based predictive control approach are accurately identified by the IDMS, at each instant of time, while the system parameters are abruptly varied with respect to time [1-11]. Based on the control strategy, the new predictive control could be so flexible to derive a highly nonlinear system in association with the traditional linear model based predictive control theory. 


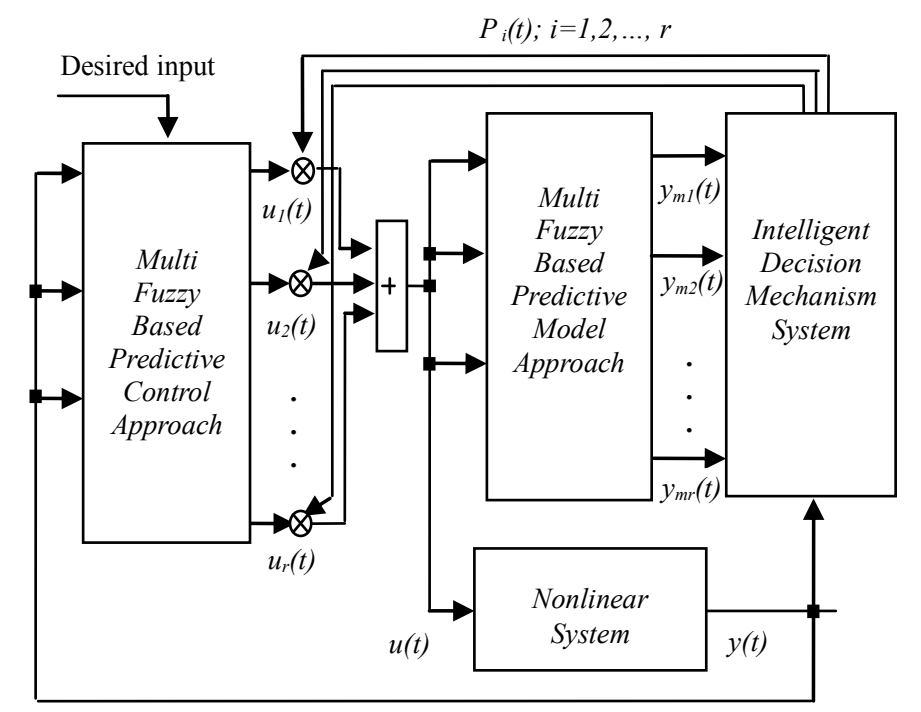

Figure 1. The scheme of the proposed control strategy

The rest of the paper is organized as follows. The proposed control scheme is given in Section 2. The simulation results and concluding remark are finally described in Sections 3 and 4, respectively.

\section{The Proposed Control Scheme}

The proposed control scheme, as shown in Figure 1 is organized based on a multi-fuzzy-based control approach as well as a multi-fuzzy-based predictive model approach and also an intelligent decision mechanism system (IDMS) to derive a severe nonlinear and time variant system. This control scheme is realized based on the predictive control theory to calculate the future control action by optimizing the specific quadratic cost function, given by

$$
J=\sum_{i=N_{1}}^{N_{2}}(y(t+i)-w(t+i))^{2}+\sum_{i=1}^{N_{u}} \lambda_{i} \Delta u(t+i-1)^{2}
$$

where $N_{u}$ is given as the control horizon, $N_{2}-N_{1}+1$ is given as the prediction horizon, $i$ is given as step ahead predictor, $y(t)$ is given as the system output, $w(t)$ is given as the desired trajectory of the future output, $\Delta u(t)$ is given as the manipulated variable and finally $\lambda$ is given as the control weight factor. In order to realize the control strategy, the number of models to cover the different operating points of the system is strongly needed and consequently the corresponding control approach needs to be designed for each one of them. If the operating points of the system are not well chosen, the outcomes corresponding to the required tracking performance cannot actually be acquired.
In the proposed control scheme, the multi-fuzzy-based predictive model approach is organized in accordance with the several fuzzy-based predictive model approaches, where each one of them is realized at a specific operating point of the system. And then the multi-fuzzybased predictive control approach is organized in accordance with the several fuzzy-based predictive control approaches, where each one of them is realized in line with the corresponding fuzzy-based predictive model approach. The proposed control scheme is an appropriate method to cope with a complex system, when we are using the predictive control theory. In fact, the proposed control scheme operates in multiple operating points, which may change from one to another abruptly. On the other hand, the operating points regions of a nonlinear and time variant system have been extended, where a linear fixed model may not really lead to the expected performance. In correspondence with the proposed control scheme, $r$ fuzzy-based predictive model approaches are used to identify the complex system at $r$ operating points and subsequently $r$ fuzzy-based predictive control approaches are used to derive the system presented at corresponding operating points. In designing the control scheme proposed, $u(t)$ is given as the control action of the multi- fuzzy-based predictive control approach, given by

$$
u(t)=\sum_{k=1}^{r} P_{k}(t) u_{k}(t)
$$

where

$$
\sum_{k=1}^{r} P_{k}(t)=1
$$


and $r$ is given as the number of the operating points of the system. Hereinafter, $P_{k}(t)$ and $u_{k}(t)$ are given as the appropriate weight and the control action of the $k^{\text {th }}$ local fuzzy-based predictive control approach, respectively.

Realization of the fuzzy based predictive control approach, the fuzzy based predictive model approach and finally the IDMS are now described in the proceeding sections.

\subsection{The Fuzzy-Based Predictive Control Approach}

The fuzzy-based predictive control approach is given as one of the local controllers in the multi-fuzzy-based predictive control approach, in the control scheme presented. The main concept in realizing the fuzzy-based predictive control approach is described by the following fuzzy rule base

$$
\begin{gathered}
\text { Rule } i: \operatorname{IF} w(t+N) \text { is } D_{j}^{i} \text { and } y_{m k}(t+N) \text { is } E_{j}^{i} \\
\text { THEN } \Delta u_{k}(t) i s F_{j}^{i}
\end{gathered}
$$

where $i$ is given as the rule number, $j$ is given as the fuzzy set number and finally $D_{j}{ }^{i}, E_{j}^{i}, F_{j}^{i}$ are given as the input-output fuzzy sets. In this control strategy, $w(t+N)$ denotes the $k^{t h}$ desired trajectory of the future output, $y_{m k}(t+N)$ denotes the fuzzy-based predictive model approach output and finally $\Delta u_{k}(t)$ denotes the manipulated variable of the $k^{\text {th }}$ fuzzy-based predictive control approach, which is directly related to the following future error signal

$$
e_{k}(t+N)=w(t+N)-y_{m_{k}}(t+N)
$$

The fuzzy set of the fuzzy-based predictive control approach is now shown in Figures 2(a) and 2(b). Moreover, the control action could easily be acquired by the following

$$
u_{k}(t)=\frac{1}{1-z^{-1}} \Delta u_{k}(t)
$$

\subsubsection{Fuzzy Sets Tuning}

In the control scheme proposed, the fuzzy sets used in realizing the multi-fuzzy-based predictive control scheme are tuned in an offline manner. In fact, the proposed tuning algorithm which is already based on the GA algorithm is realized for all the fuzzy-based predictive control approaches. With this purpose, these fuzzy sets are first parameterized, as shown in Figure 3 using $a_{i}, b_{i}, c_{i}$, $d_{j} ; i=1,2,3, j=1,3$. In this way, some populations are chosen, while the first population has nine chromosomes $\left(V_{j} ; j=1,2, \ldots, 9\right)$, which is now realized by the following forms

$$
\begin{aligned}
& V_{j}= \\
& \left\{g_{1 j}, g_{2 j}, g_{3 j}, g_{4 j}, g_{5 j}, g_{6 j}, g_{7 j}, g_{8 j}, g_{9 j}, g_{10 j}, g_{11 j}\right\}
\end{aligned}
$$

where we could have the following

$$
\left\{\begin{array}{lll}
g_{1 j}=a_{1}+\delta_{a_{1 j}} & g_{2 j}=a_{2}+\delta_{a_{2 j}} & g_{3 j}=a_{3}+\delta_{a_{3 j}} \\
g_{4 j}=b_{1}+\delta_{b_{1 j}} & g_{5 j}=b_{2}+\delta_{b_{2 j}} & g_{6 j}=b_{3}+\delta_{b_{3 j}} \\
g_{7 j}=c_{1}+\delta_{c_{1 j}} & g_{8 j}=c_{2}+\delta_{c_{2 j}} & g_{9 j}=c_{3}+\delta_{c_{3 j}} \\
g_{10 j}=d_{1}+\delta_{d_{1 j}} & g_{11 j}=d_{3}+\delta_{d_{3 j}} &
\end{array}\right.
$$

In such a way, with a specific mutation rate; $P c=0.6$, the $2^{\text {nd }}$ population have five chromosomes and the $3^{\text {rd }}$ population have three chromosomes. Also the $4^{\text {th }}$ population has two chromosomes and the $5^{\text {th }}$ populations have

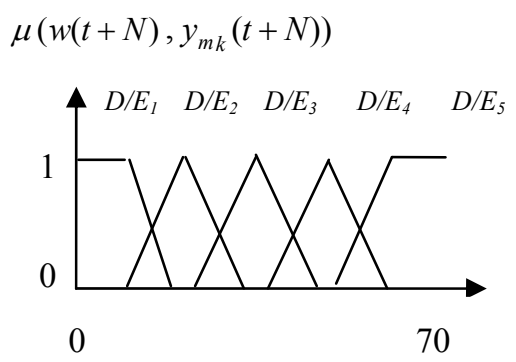

(a)

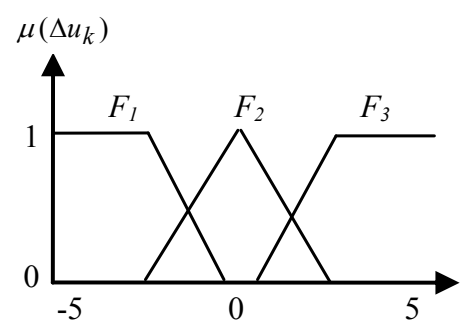

(b)

Figure 2. (a) The input fuzzy sets used in the fuzzybased predictive control approach; (b) The output fuzzy sets used in the fuzzy-based predictive control approach

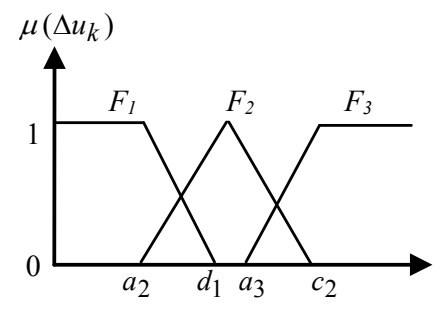

Figure 3. The fuzzy sets used in the tuning algorithm 
one chromosome. Obviously, the fuzzy sets parameters could be optimized to appropriate values by the superior genes.

\subsection{The Fuzzy-Based Predictive Model Approach}

The fuzzy-based predictive model approach is realized as one of models in the multi-fuzzy-based predictive model approach. In case of this approach, $u(t-\tau)$ is given as a valid input of the system, while $\tau$ is given as its delay. Also, $y_{m k}(t)$ is given as the $k^{t h}$ fuzzy-based model output. The schematic of this approach is shown in Figure 4 . Here, the fuzzy-based model\#N could be placed in sequence with the fuzzy-based model\#(N-1) to provide the fuzzy-based predictive model approach. In fact, the prediction of model output could be improved, while the number of the present fuzzy-based models is increased. The fuzzy rule based for this approach is now given as follows

$$
\begin{gathered}
\text { Rule } i: \operatorname{IF} u(t-\tau) \text { is } A_{j}{ }^{i} \text { and } y_{m k}(t-1) \text { is } B_{j}^{i} \\
\text { THEN } y_{m k}(t) i s C_{j}^{i}
\end{gathered}
$$

where $i$ is given as the rule number, $j$ is given as the fuzzy set number and finally $A_{j}{ }^{i}, B_{j}^{i}, C_{j}^{i}$ are given as the input-output fuzzy sets, as shown in Figure 5.

\subsection{The IDMS Realization}

In the control scheme proposed, the best fuzzy-based predictive model approach in the multi-fuzzy-based predictive model approach is accurately identified by using the IDMS, at each instant of time. Now, by assuming only three fuzzy-based predictive model approaches, for simplicity, the IDMS can be summarized as follows

- Defining the specific performance indices; $J_{i}(t) ; i=$ $1,2,3$, for each one of the fuzzy-based predictive model approaches, correspondingly, given by

$$
\begin{array}{r}
J_{i}(t)=\alpha e_{i}^{2}(t)+\beta \int_{0}^{t} e^{-\lambda(t-\tau)} e_{i}^{2}(\tau) d \tau \\
; \alpha \geq 0 ; \beta, \lambda>0
\end{array}
$$

where

$$
e_{i}(t)=y(t)-y_{i}(t)
$$

In the IDMS proposed, $\alpha, \beta$ and $\lambda$ are given as the weighting factors on the instantaneous measures, the long term accuracy and the forgetting factor, respectively.
- Defining the input-output fuzzy sets, as shown in Figure 6.

- Defining the fuzzy rules based, which can be given by the following

Rule\#1:

$$
\begin{aligned}
& \text { IF } J_{1}(t) \text { is } S_{J}^{1} A N D J_{2}(t) \text { is } L_{J}^{1} A N D J_{3}(t) \text { is } L_{J}^{1} \\
& \text { THEN } P_{1}(t) \text { is } L_{P}^{1}
\end{aligned}
$$

Rule\#2:

$$
\begin{aligned}
& \text { IF } J_{1}(t) \text { is } L_{J}^{2} \text { AND } J_{2}(t) \text { is } S_{J}^{2} \text { AND } J_{3}(t) \text { is } L_{J}^{2} \\
& \text { THEN } P_{2}(t) \text { is } L_{P}^{2}
\end{aligned}
$$

Rule\#3:

$$
\begin{aligned}
& \text { IF } J_{1}(t) \text { is } L_{J}^{3} \text { AND } J_{2}(t) \text { is } L_{J}^{3} \text { AND } J_{3}(t) \text { is } S_{J}^{3} \\
& \text { THEN } P_{3}(t) \text { is } L_{P}^{3}
\end{aligned}
$$

\section{Simulation Results}

In order to verify the applicability of the proposed control scheme so called MFPMPC scheme in this paper, an industrial tubular heat exchanger system is used to control [1-11]. To analyze the proposed control scheme in comparison with other control techniques which are fully investigated by the Authors in the several publications, this complex system is realized the same specification as these research works, where all the research papers could easily be found by the readers in detail.

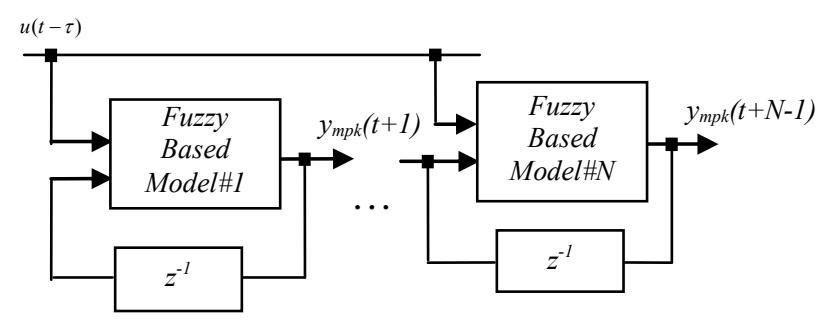

Figure 4. The scheme of the fuzzy-based predictive model approach

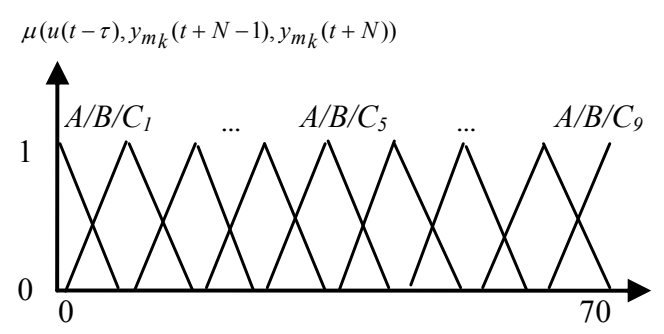

Figure 5. The fuzzy sets used in fuzzy-based predictive model approach 

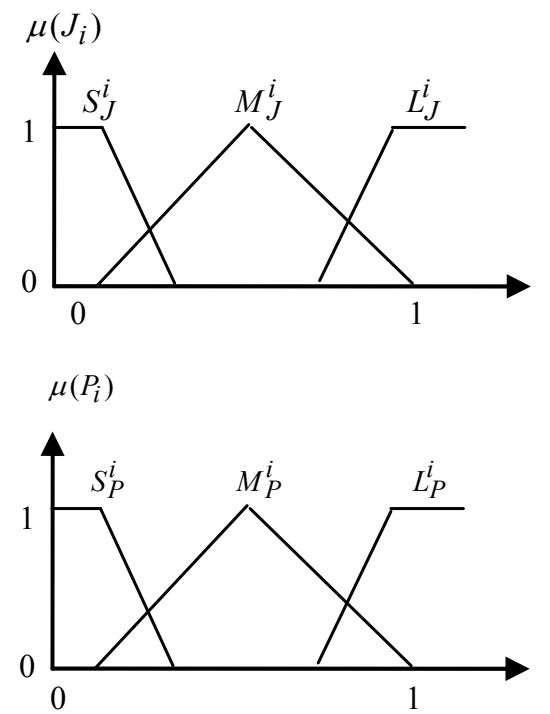

Figure 6. The fuzzy sets used in the IDMS

With this purpose, we have carried out the MFPMPC scheme for this system using the Simulink-Matlab programming language, while the multi-fuzzy-based predictive model approach is organized based on the three fuzzy-based predictive model approaches and subsequently the multi-fuzzy-based predictive control approach is correspondingly organized based on the three fuzzy-based predictive model approaches. In these simulations, the tracking performance of the MFPMPC scheme is given in Figure 7, while the single model linear generalized predictive control entitled SLMGPC scheme is used as a benchmark approach here. At first, the desired trajectory of the future output is given as $60^{\circ} \mathrm{C}$ at $5 \mathrm{sec}$., while it is abruptly varied to $55^{\circ} \mathrm{C}$ and also $40^{\circ} \mathrm{C}$ at $54 \mathrm{sec}$. and $104 \mathrm{sec}$., respectively. In these simulations, the SLMGPC scheme is carried out by the following control parameters

$$
\left\{\begin{array}{l}
N_{2}-N_{1}+1=3 \\
N_{u}=3
\end{array}\right.
$$

And the CARIMA model is also given by

$$
\begin{aligned}
& A\left(q^{-1}\right) y(k)=B\left(q^{-1}\right) u(k-1)+\frac{e(k)}{\Delta\left(q^{-1}\right)} \\
& A\left(q^{-1}\right)=1+a_{1} q^{-1}+\ldots+a_{p} q^{-p} ; p=4 \\
& B\left(q^{-1}\right)=b_{0}+b_{1} q^{-1}+\ldots+b_{m} q^{-m} ; m=p
\end{aligned}
$$

where its coefficients are identified using the RLS algorithm, as tabulated in Table 1. Afterwards, the weight signals which are all resulted from the proposed IDMS is shown in Figure 8. In such a case, the multi-fuzzy-based

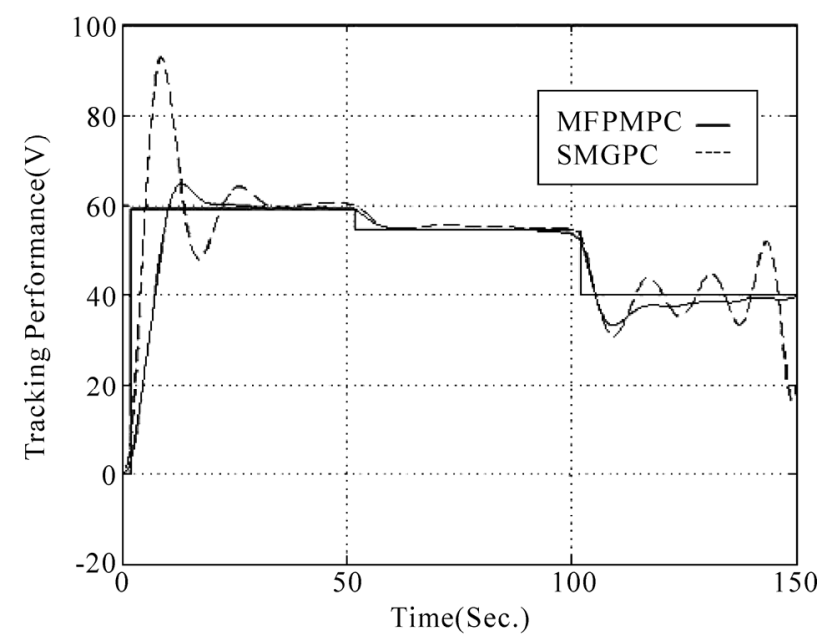

Figure 7. The scheme of MFPMPC scheme tracking performance in comparison with the SLMGPC scheme

predictive model approach outputs are given in Figure 9 as well. Hereinafter, the manipulated variable signals and the corresponding control actions are all given in Figures 10 and 11 , respectively. Here the control actions are all multiplied by its weights, in these figures. As is easily obvious from the outcomes, the tracking performance of the MFPMPC scheme is quite outperformed with respect to its benchmark approach.
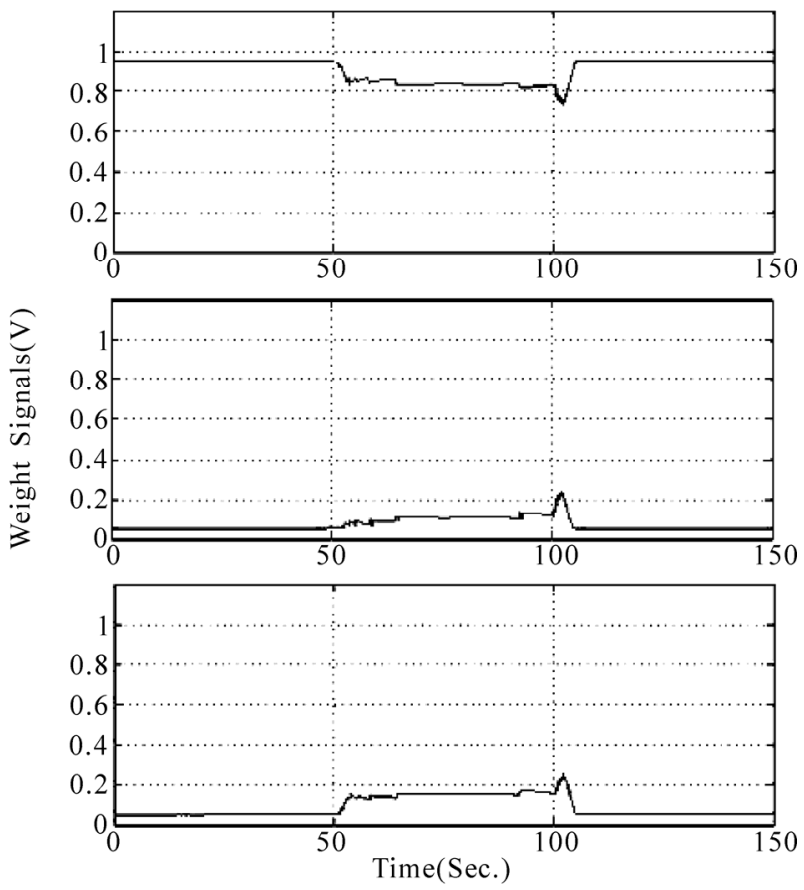

Figure 8. The scheme of the MFPMPC weight signals, given by the proposed IDMS [The first diagram is related to $P_{1}(t)$, the second diagram is related to $P_{2}(t)$ and the third diagram is finally related to $\left.P_{3}(t)\right]$ 

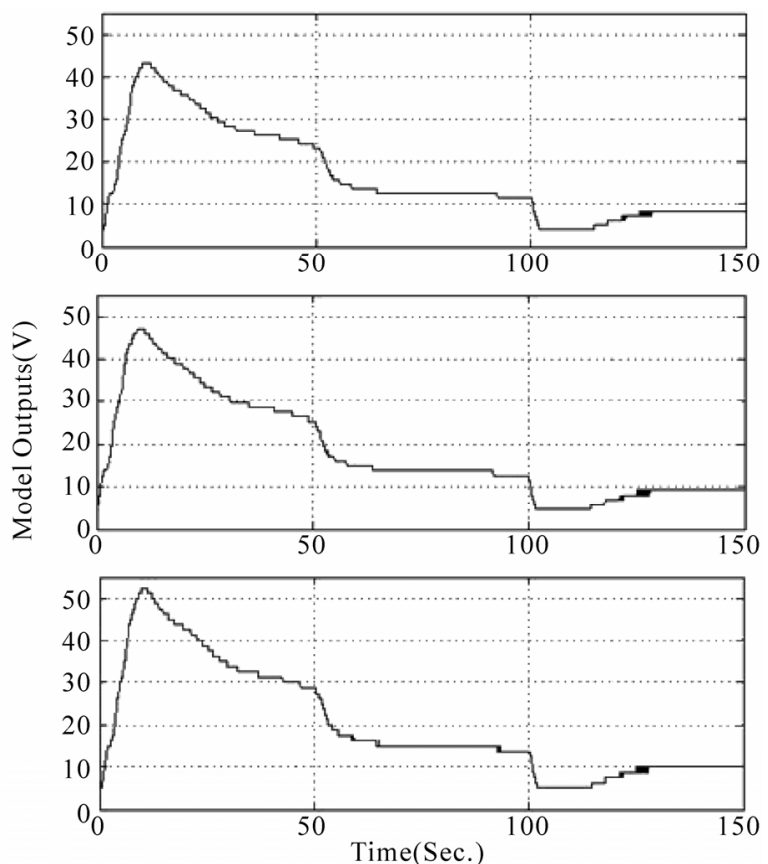

Figure 9. The scheme of the MFPMPC models outputs, given by the multi-fuzzy-based predictive model approach [The first diagram is related to $y_{m I}(t)$, the second diagram is related to $y_{m 2}(t)$ and the third diagram is finally related to $\left.y_{m 3}(t)\right]$

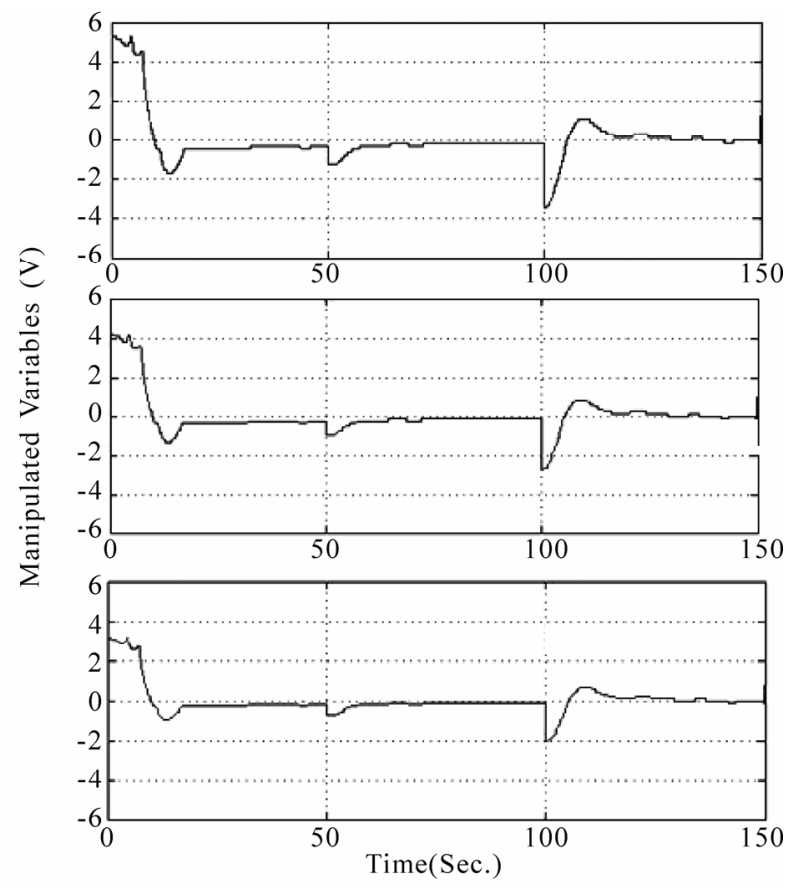

Figure 10. The scheme of the MFPMPC manipulated variables [The first diagram is related to $\Delta u_{1}(t)$, the seconddiagram is related to $\Delta u_{2}(t)$ and the third diagram is finally related to $\left.\Delta u_{3}(t)\right]$
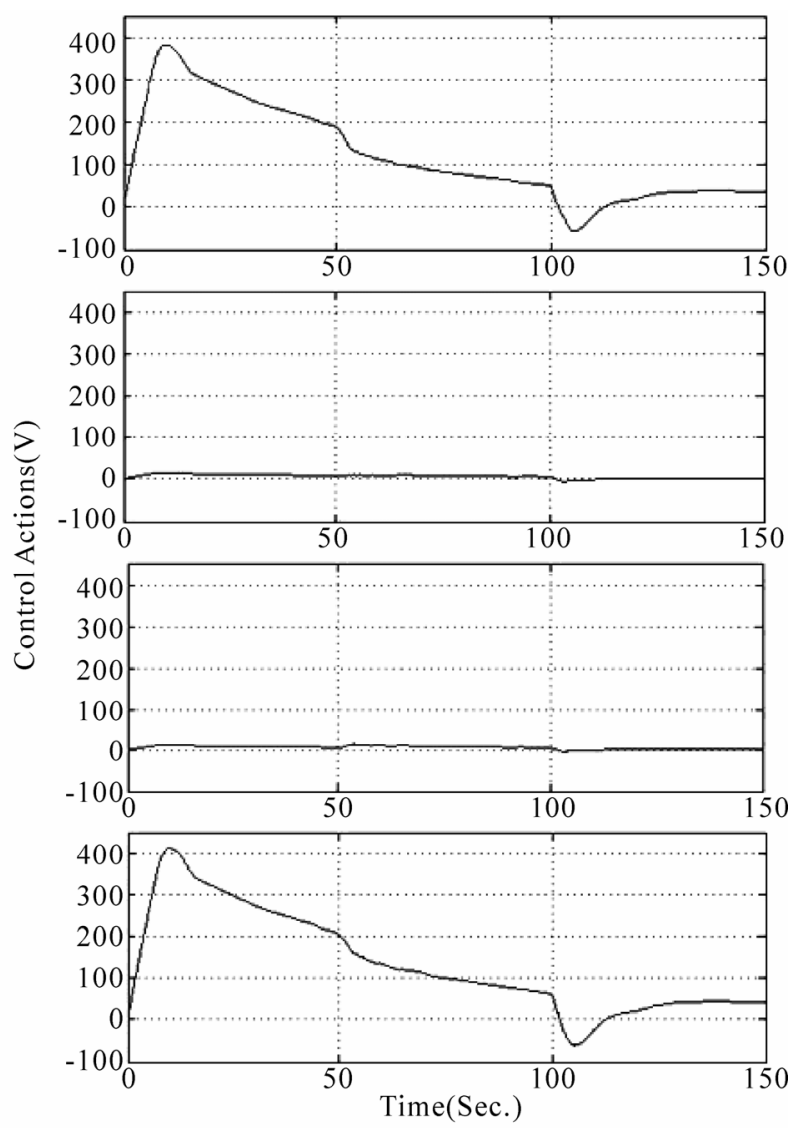

Figure 11. The scheme of the MFPMPC control actions [The first diagram is related to $u_{1}(t)$, the second diagram is related to $u_{2}(t)$, the third diagram is related to $u_{3}(t)$ and finally the fourth diagram is finally related to $u(t)$ ]

\section{Conclusions}

A main problem in realizing the linear model based predictive control theory to derive a severe nonlinear system has been thoroughly solved, in this paper. Based on the proposed control strategy so called MFMPMC scheme by the Authors, a nonlinear system must first be represented via the multi-fuzzy-based predictive model approach. And then the multi-fuzzy-based predictive control approach is correspondingly realized to derive the nonlinear system presented.

Table 1. The coefficients of the CARIMA model

\begin{tabular}{ccc}
\hline$j$ & $a_{j}$ & $b_{j}$ \\
\hline 1 & -0.9933 & $0.2506 \mathrm{e}-3$ \\
2 & -0.4342 & $0.3519 \mathrm{e}-3$ \\
3 & 0.0069 & $0.5283 \mathrm{e}-3$ \\
4 & 0.4219 & $0.1830 \mathrm{e}-3$ \\
\hline
\end{tabular}


Besides, the intelligent decision mechanism system (IDMS) is used to identify the best fuzzy-based predictive model approach and the corresponding fuzzy-based predictive control approach, at each instant of time. The advantage of the proposed control scheme over other related control techniques is to realize the strategy in the absence of any linear model approximation of the complex system. It points out that the proposed MFPMPC scheme is the generalized version of the traditional model based predictive control theory. Also the present control structure aims us to develop it for all the highly nonlinear and time variant systems in both real and academic environments.

\section{References}

[1] A. H. Mazinan and M. F. Kazemi, "An efficient solution to load - frequency control using fuzzy - based predictive scheme in a two-area interconnected power system," The 2nd International Conference on Computer and Automation Engineering, 2010.

[2] A. H. Mazinan and N. Sadati, "An intelligent multiple models based predictive control scheme with its application to industrial tubular heat exchanger system," Applied Intelligence, Springer Publisher, DOI 10.1007/s10489009-0185-8, in press, 2009.

[3] A. H. Mazinan and N. Sadati, "Fuzzy predictive control based multiple models strategy to a tubular heat exchanger system," Applied Intelligence, Springer Publisher, DOI 10.1007/s10489-009-0163-1, in press, 2009.

[4] A. H. Mazinan and N. Sadati, "On the application of fuzzy predictive control based on multiple models strategy to a tubular heat exchanger system," Transactions of the Institute of Measurement \& Control, SAGE Publisher, DOI 10.1177/0142331209345153, in press, 2009.

[5] A. H. Mazinan and A. H. Hosseini, "Application of intelligent based predictive scheme to load-frequency control in a two-area interconnected power system," Applied Intelligence, in press, 2009.

[6] A. H. Mazinan and N. Sadati, "A comparative study on applications of artificial intelligence based multiple models predictive scheme to industrial tubular heat exchanger system," ISA Transactions, Elsevier Publisher, in press, 2009.

[7] A. H. Mazinan, N. Sadati, and H. Ahmadi-Noubari, "A case study for fuzzy adaptive multiple models predictive control strategy," in Proc. of IEEE World Symposium on Industral Electronics, pp. 1172-1177, 2009.

[8] A. H. Mazinan and N. Sadati,'Fuzzy multiple models predictive control of tubular heat exchanger," in Proc. of IEEE World Congress on Computational Intelligence, pp. 1845-1852, 2008.

[9] A. H. Mazinan and N. Sadati, "Multiple modeling and fuzzy predictive control of a tubular heat exchanger system," Transactions on Systems and Control, Vol. 3, pp. 249-258, 2008

[10] A. H. Mazinan and N. Sadati, "Fuzzy multiple modeling and fuzzy predictive control of a tubular heat exchanger system," International Conference on Application of Electrical Engineering, pp. 77-81, 2008.

[11] A. H. Mazinan and N. Sadati, "Fuzzy multiple modeling and fuzzy predictive control of a tubular heat exchanger system," International Conference on Robotics, Control and Manufacturing Technology, China, pp. 93-97, April 2008. 